\title{
Evidence for a novel coding sequence overlapping the 5'-terminal 90 codons of the Gill-associated and Yellow head okavirus envelope glycoprotein gene
} Andrew E Firth*1 and John F Atkins*1,2

\author{
Address: ${ }^{1 B i o S c i e n c e s ~ I n s t i t u t e, ~ U n i v e r s i t y ~ C o l l e g e ~ C o r k, ~ C o r k, ~ I r e l a n d ~ a n d ~}{ }^{2}$ Department of Human Genetics, University of Utah, Salt Lake City, \\ UT 84112-5330, USA \\ Email: Andrew E Firth* - A.Firth@ucc.ie; John F Atkins* - j.atkins@ucc.ie \\ * Corresponding authors
}

Published: 17 December 2009

Virology Journal 2009, 6:222 doi:10.1 186/1743-422X-6-222

This article is available from: http://www.virologyj.com/content/6/1/222

(c) 2009 Firth and Atkins; licensee BioMed Central Ltd.

This is an Open Access article distributed under the terms of the Creative Commons Attribution License (http://creativecommons.org/licenses/by/2.0), which permits unrestricted use, distribution, and reproduction in any medium, provided the original work is properly cited.

\begin{abstract}
The genus Okavirus (order Nidovirales) includes a number of viruses that infect crustaceans, causing major losses in the shrimp industry. These viruses have a linear positive-sense ssRNA genome of $\sim 26-27 \mathrm{~kb}$, encoding a large replicase polyprotein that is expressed from the genomic RNA, and several additional proteins that are expressed from a nested set of 3 '-coterminal subgenomic RNAs. In this brief report, we describe the bioinformatic discovery of a new, apparently coding, ORF that overlaps the 5 ' end of the envelope glycoprotein encoding sequence, ORF 3 , in the +2 reading frame. The new ORF has a strong coding signature and, in fact, is more conserved at the amino acid level than the overlapping region of ORF3. We propose that translation of the new ORF initiates at a conserved AUG codon separated by just $2 \mathrm{nt}$ from the ORF3 AUG initiation codon, resulting in a novel 86 amino acid protein.
\end{abstract}

\section{Findings}

The genus Okavirus belongs to the family Roniviridae in the order Nidovirales. Members include Gill-associated virus (GAV) and Yellow head virus (YHV), both of which infect Penaeus monodon shrimp. GAV and YHV are currently classified as two of six or more genotypes of the single species Gill-associated virus or the Yellow head complex. As with other members of the order Nidovirales, these viruses have a linear positive-sense ssRNA genome encoding a large replicase polyprotein that is expressed from the genomic RNA (ORF1a and, via ribosomal frameshifting, an ORF1aORF1b fusion product), and a number of other proteins including the structural proteins - which are translated from a nested set of 3'-coterminal sub-genomic RNAs [1$4]$. In the case of the okaviruses, the 3 ' ORFs encode a polyprotein (ORF3; 1650 codons) that is cleaved to pro- duce three mature proteins (envelope glycoproteins gp116 and gp64, and an 25 kDa N-terminal fragment), a nucleocapsid (ORF2; 145 codons), and possibly an additional short ORF (ORF4) encoded by the sequence 3' of ORF3 (Figure 1A) [4-10].

Overlapping genes are common in RNA viruses where they serve as a mechanism to optimize the coding potential of compact genomes. However, annotation of overlapping genes can be difficult using conventional genefinding software [11]. Recently we have been using a number of complementary approaches to systematically identify new overlapping genes in virus genomes [11-15]. When we applied these methods to the okaviruses, we found strong evidence for a new coding sequence - hereafter ORFX - overlapping the 5'-terminal 88 codons of ORF3 


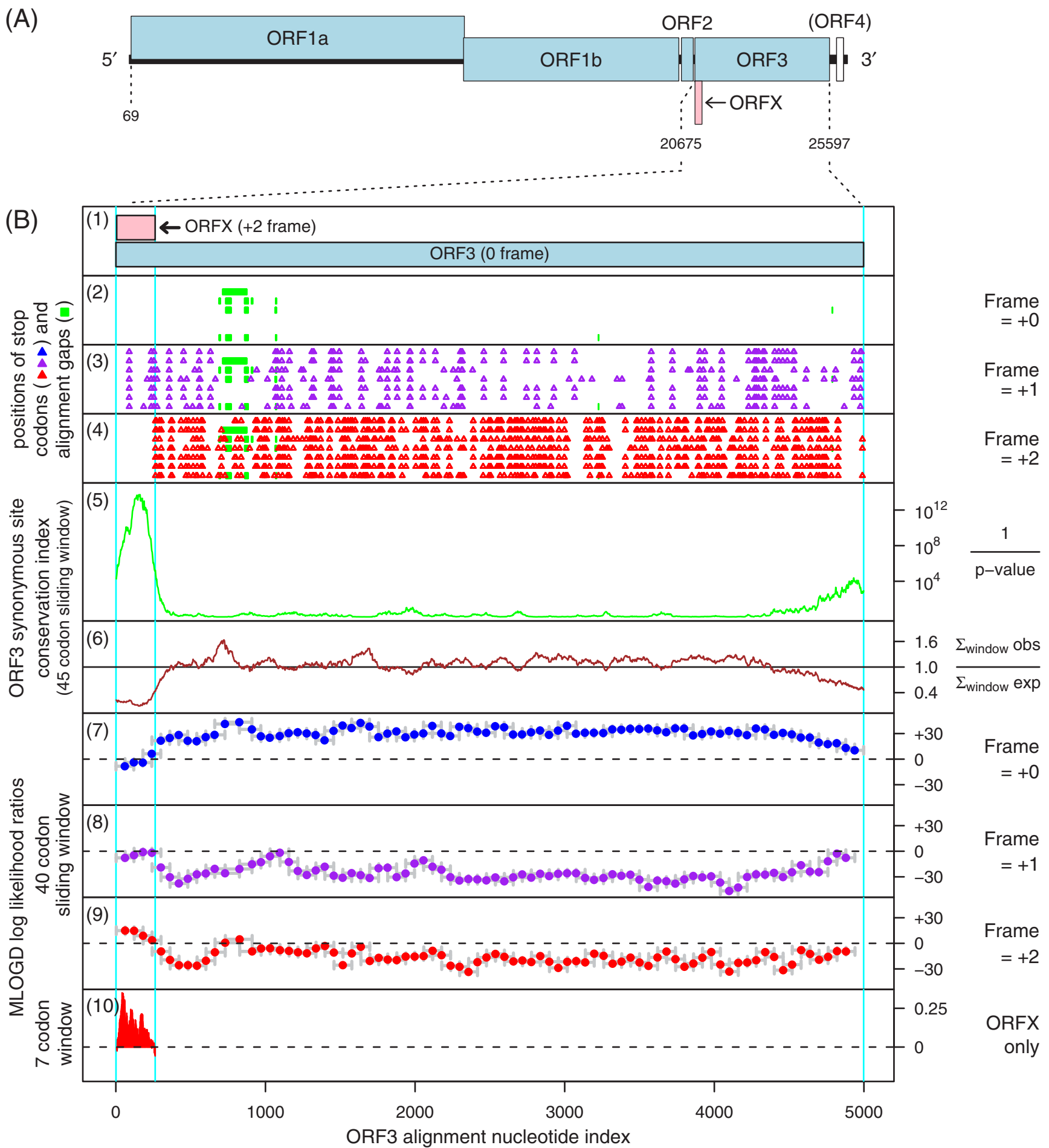

Figure I (see legend on next page) 
Figure I (see previous page)

Coding potential statistics for okavirus ORF3 and the overlapping ORFX. (A) Okavirus genome map (GAV [GenBank:AF227|96]). (B2-B I 0) Coding potential statistics based on an alignment of seven okavirus full-length ORF3 sequences (see text for accession numbers). (B2-B4) Positions of stop codons in each of the three forward reading frames. Note the conserved absence of stop codons in the +2 frame within ORFX. (B5-B6) Conservation at synonymous sites within ORF3 (see [15]). (B5) depicts the probability that the degree of conservation within a given window could be obtained under a null model of neutral evolution at synonymous sites, while (B6) depicts the ratio of the observed number of substitutions within a given window to the number expected under the null model. (B7-B9) MLOGD sliding-window plots (see [I2]). The null model, in each window, is that the sequence is non-coding, while the alternative model is that the sequence is coding in the given reading frame. Positive scores favour the alternative model and, as expected, there is a strong coding signature in the +0 frame (B7) throughout ORF3 except where ORF3 is overlapped by ORFX. In the $+I$ and +2 frames (B8-B9), scores are generally negative. However, the ORFX region has consecutive high positively scoring windows (B9). (B I 0) MLOGD statistics restricted to ORFX. Here, for increased sensitivity, the null and alternative models were fitted specifically for the ORFX region. The null model is that only the ORF3 frame is coding, while the alternative model is that both the ORF3 frame and ORFX are coding.

in the +2 reading frame (Figure 1 ). Here we describe the bioinformatic analyses.

Okavirus sequences in GenBank with full-length coverage of ORF3 were identified by applying tblastn [16] to the ORF3 amino acid sequence from one GAV isolate [GenBank:AF227196] and one YHV isolate [GenBank:EU487200], resulting in the additional sequences [GenBank:EF156405], [GenBank:F[848673], [GenBank:FJ194949], [GenBank:EU785042] and [GenBank:EU785043]. The ORF3 regions were extracted, translated, aligned with CLUSTALW [17], and back-translated to give a nucleotide sequence alignment for ORF3.

The alignment was analysed for conservation at synonymous sites, as described in [15]. The procedure takes into account whether synonymous site codons are 1-, 2-, 3-, 4or 6-fold degenerate and the differing probabilities of transitions and transversions. The analysis revealed a striking, and highly statistically significant $\left(p<10^{-21}\right.$ for the total conservation within ORFX), peak in synonymous site conservation at the 5 ' end of ORF3 (Figure 1B, panels 5-6). Such conservation peaks are indicative of overlapping functional elements, though such elements may be either coding or non-coding. However, in this case, coinciding with the conserved region there was a conserved absence of stop codons in the +2 reading frame (Figure $1 \mathrm{~B}$; panel 4), thus suggesting an overlapping coding sequence in the +2 frame as a possible explanation for the enhanced conservation at ORF3-frame synonymous sites.

Inspection of an additional 33 sequences (FJ438530FJ438532, FJ428584-FJ428613; [18]) with only partial coverage of ORF3, but nearly complete coverage of the ORFX region, again revealed the complete absence of +2 frame stop codons in ORFX. One further partial sequence, [GenBank:DQ978360], differed in having a single nucleotide deletion $~ 15$ codons into ORFX. The effect of this deletion is to fuse the $5^{\prime}$ end of ORFX with the downstream ORF3: ribosomes initiating at the ORF3 initiation codon terminate early while ribosomes initiating at the ORFX initiation codon (see below) go on to translate an ORFX-ORF3 fusion. In fact, except for this deletion, the $717 \mathrm{nt}$ DQ978360 is identical to the corresponding region of FJ848673, so it is possible that this deletion is simply due to a sequencing error or a defective sequence.

Given the short length of ORFX, conservation alone is perhaps not sufficient evidence for a coding assignment. Therefore, the alignment was also analysed with MLOGD - a gene-finding program which was designed specifically for identifying overlapping coding sequences, and which includes explicit models for sequence evolution in multiply-coding regions $[11,12]$. In contrast to the synonymous site conservation index above, MLOGD, when applied in the 'sliding window' mode, does not depend on the degree of conservation per se (the sequence divergence parameter is fitted independently for each window). When applied to the ORF3 alignment, MLOGD detected a strong coding signature for ORFX - with positively scoring windows throughout the ORFX region in the +2 frame - indicating directly that ORFX is indeed a coding sequence (Figure 1B, panel 9). In fact, the MLOGD score in the +2/ORFX frame within the ORFX region was significantly greater than the score in the +0/ORF3 frame, indicating that the ORFX product is subject to stronger functional constraints than the product of the overlapping region of ORF3 (which indeed has a negative MLOGD score towards the 5 '-terminal half of the ORFX region; Figure 1B, panel 7). Consistently, a comparison of GAV AF227196 with YHV EU487200 showed that, in the region where ORFX and ORF3 overlap, ORFX has higher amino acid conservation than ORF3 (71/86 identities for ORFX, 62/86 identities for ORF3). 
When MLOGD was applied in the 'test query ORF' mode (Figure 1B, panel 10), the number of independent base variations across the alignment within the ORFX region was calculated to be $\mathrm{N}_{\mathrm{var}} \sim 57$, and the total MLOGD score was $\log (\mathrm{LR}) \sim 33.0$ (see [12] for details). Although MLOGD has a significant false negative rate (i.e. there are known overlapping genes that it fails to detect - particularly ones that are less conserved than the genes they overlap), the false positive rate (with appropriate thresholds) is low. In particular, extensive tests with known singlecoding and double-coding virus sequence alignments indicate that ' $\mathrm{N}_{\mathrm{var}} \geq 20$ ' and ' $\log (\mathrm{LR}) \geq \frac{1}{6} \times \mathrm{N}_{\mathrm{var}}$ ' signals robust detection ( $<1 \%$ false positive rate) of an overlapping same-strand CDS [12] (and unpublished data).

As of 27 Sep 2009, we located 11 okavirus sequences with coverage of the ORF3 initiation codon, 10 of which also included coverage of the 35 nt ORF3 sgRNA 5'UTR [8]. Inspection of these sequences showed that all 11 possessed a +2/ORFX-frame AUG codon downstream of, and separated by just 2 nt from, the ORF3 AUG initiation codon (Figure 2A). Both the ORF3-frame and ORFXframe AUG codons generally have a ' $G$ ' at -3 and a pyrimidine at +4 , resulting in a medium rather than strong Kozak context. In Turnip yellow mosaic tymovirus, the AUG initiation codons of the overlapping p69 and p206 coding sequences are also closely spaced (4 nt separa- tion). In this case, it has been demonstrated with mutant sequences that, provided the two AUG codons are separated by not more than $\sim 10 \mathrm{nt}$, the efficiency of initiation at the second AUG codon is greatly enhanced compared with the efficiency obtained for canonical leaky scanning (i.e. for greater separations) given the same Kozak contexts [19]. Moreover, initiation is competitively coupled in the sense that mutation of the downstream AUG codon increases the efficiency of initiation at the upstream AUG codon, and vice versa. Thus, initiation at the okavirus ORFX-frame AUG codon is likely to be significantly more efficient than would be expected for normal leaky scanning. Only two of the 11 okavirus sequences have additional upstream AUG codons (Figure 2A). EU785043 has an additional ORF3-frame AUG codon two codons upstream - apparently due to a duplication of the nucleotides 'AUGCAA'. This does not affect our interpretation, however, since the proximity of the ORFX-frame AUG codon to the first ORF3-frame AUG codon (8 nt separation) is within the bounds for the translation initiation coupling mechanism of Ref. [19]. EU785042, on the other hand, has an apparent duplication of the nucleotides 'AUGCA', resulting in an upstream AUG codon in-frame with neither ORF3 nor ORFX. Again all three AUG codons are separated by no more than $7 \mathrm{nt}$, thus presumably allowing efficient initiation on all three; however, the total number of ribosomes translating ORF3 and ORFX will be depleted due to those that initiate on the first AUG codon (31 codon ORF).

\title{
(A)
}
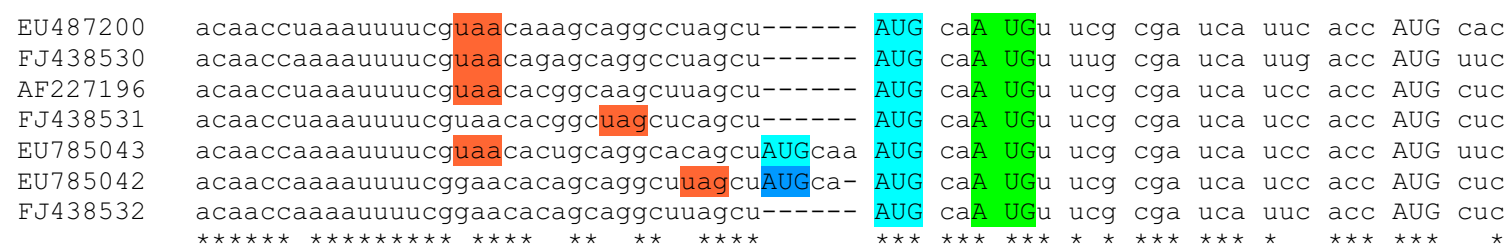

genotype 1 genotype 5 genotype 2 genotype 2 genotype 4 genotype 3 genotype 3

(B)

EU4 87200 FJ4 38530 AF227196 FJ438531 EU785043 EU785042 FJ438532

\begin{abstract}
MFAI IHHALLILSLPAIFIFYWAAGLISDLPLIYFTLWFWGSWLLATICYSLTFSGRRRNKDKFYYS INVQQHNPYNKRILSSKFA MFA I IDHVLLVLTLPS LF IFYWAAGLISDLPLIYLTLWLWGSWLLSTICYSLTFGGRRRNKDKFYYS INVHQHNPYSKRIMSSKLA MFA I I HHALLLLTLPSLI I FYWAAGY ISDLPLVFSTLWIWGSWLCTT ICYSLTFSGRRRNKDKFYYS INVQQHNPYSKRIMSSKLA MFA I IHHALLLLTLPSLI I FYWAAGY I SDLPLVFSTLWIWGSWLCTTICYSLTFSGRRRNKDKFYYS INVQOHNPYSKRIMSSKLA MFAI I HHVLLFLSLPSLI I FYWAAGY ISDLPLVLSTLWIWGSWLCTTICYSLTFSGRRRNKDKFYYS INVQQHNPYSRRILSSKLA MFA I IHHALLLITLPSLLIFYWAAGY ISDLPLVLSTLWIWGSWLCTTICYTLTFSGRRRNKDKFYYS INVOOHNPHSKRILSSKLA MFA I IHHALLLLTLPSLLIFYWAAGY ISDLPLVLSTLWIWGSWLCTT ICYTLTFSGRRRNKDKFYYS INVQQHNPHSKRILSSKLA

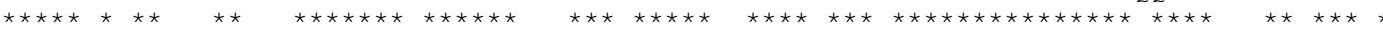

\section{Figure 2}

Nucleotide and amino acid sequence alignments. (A) Nucleotide alignment of the 5'-terminal region of the ORF3 sgRNA. All currently available and non-identical okavirus sequences with coverage of this region are shown. A further four sequences (DQ978360, FJ194949, FJ848673 and EFI56405) are locally identical to EU487200. Genotype designations within the Yellow head complex are based on the ORF3 phylogeny of Ref. [I8]. Spaces separate +0/ORF3-frame codons. All AUG codons are indicated in capitals. Colour coding is as follows: light blue - ORF3 initiation codon(s); green - proposed +2/ORFXframe initiation codon; dark blue - upstream + I frame AUG codon (3 I codon ORF); orange - last upstream ORFX-frame stop codons. (B) Amino acid alignment of the translated ORFX for representative okavirus sequences. 
Initiation at the proposed AUG codon would give ORFX the nucleotide coordinates 20680..20937 in AF227196 (GAV) and 20990..21247 in EU487200 (YHV), resulting in an 86 amino acid product with a molecular mass of 10 $\mathrm{kDa}$. The full predicted amino acid sequences are shown in Figure 2B. Application of blastp [16] to the amino acid sequences revealed no similar sequences in GenBank - as expected for a gene created de novo via out-of-frame 'overprinting' of a preexisting gene [20,21]. Application of InterProScan [22] predicted an N-terminal signal peptide/ transmembrane region, and an additional transmembrane region comprising amino acids 34 to 54. Application of SignalP 3.0 [23] predicted an $\mathrm{N}$-terminal signal anchor or signal peptide. If the latter, cleavage of the signal peptide - mostly probably between residues 25 and 26 - would leave a 61 amino acid, $7.3 \mathrm{kDa}$ ORFX product.

Overlapping genes are difficult to identify and are often overlooked. However, it is important to be aware of such genes as early as possible in order to avoid confusion (otherwise functions of the overlapping gene may be wrongly ascribed to the gene they overlap), and also so that the functions of the overlapping gene may be investigated in their own right. We hope that presentation of this bioinformatic analysis will help fullfil these goals. Initial verification of ORFX product could be by means of immunoblotting with ORFX-specific antibodies.

\section{Competing interests}

The authors declare that they have no competing interests.

\section{Authors' contributions}

AEF carried out the bioinformatic analysis and wrote the manuscript. Both authors edited and approved the final manuscript.

\section{Acknowledgements}

This work was supported by National Institutes of Health Grant R0 I GM079523 and an award from Science Foundation Ireland, both to JFA.

\section{References}

I. Cowley JA, Dimmock CM, Walker PJ: Gill-associated nidovirus of Penaeus monodon prawns transcribes 3'-coterminal subgenomic mRNAs that do not possess 5'-leader sequences. J Gen Virol 2002, 83:927-935.

2. Gorbalenya AE, Enjuanes L, Ziebuhr J, Snijder EJ: Nidovirales: evolving the largest RNA virus genome. Virus Res 2006, I | 7:17-37.

3. Pasternak AO, Spaan WJ, Snijder EJ: Nidovirus transcription: how to make sense...? J Gen Virol 2006, 87:|403-I42I.

4. Sittidilokratna N, Dangtip S, Cowley JA, Walker PJ: RNA transcription analysis and completion of the genome sequence of yellow head nidovirus. Virus Res 2008, I36: I57-I65.

5. Cowley JA, Walker PJ: The complete genome sequence of gillassociated virus of Penaeus monodon prawns indicates a gene organisation unique among nidoviruses. Arch Virol 2002, | 47:1977-1987.

6. Jitrapakdee S, Unajak S, Sittidilokratna N, Hodgson RA, Cowley JA, Walker PJ, Panyim S, Boonsaeng V: Identification and analysis of gp I 16 and gp64 structural glycoproteins of yellow head nidovirus of Penaeus monodon shrimp. J Gen Virol 2003, 84:863-873.
7. Cowley JA, Cadogan LC, Spann KM, Sittidilokratna N, Walker PJ: The gene encoding the nucleocapsid protein of Gill-associated nidovirus of Penaeus monodon prawns is located upstream of the glycoprotein gene. J Virol 2004, 78:8935-894I.

8. Wijegoonawardane PK, Cowley JA, Phan T, Hodgson RA, Nielsen L, Kiatpathomchai W, Walker PJ: Genetic diversity in the yellow head nidovirus complex. Virology 2008, 380:2I3-225.

9. Sittidilokratna $\mathrm{N}$, Chotwiwatthanakun C, Wijegoonawardane PK, Unajak S, Boonnad A, Wangnai W, Jitrapakdee S, Cowley JA, Walker PJ: A virulent isolate of yellow head nidovirus contains a deformed envelope glycoprotein gpl I6. Virology 2009 , 384:192-200.

10. Gangnonngiw W, Anantasomboon G, Sang-oum W, Sriurairatana S, Sritunyalucksana K, Flegel TW: Non-virulence of a recombinant shrimp nidovirus is associated with its non structural gene sequence and not a large structural gene deletion. Virology 2009, 385:16I-168.

II. Firth $\mathrm{AE}$, Brown $\mathrm{CM}$ : Detecting overlapping coding sequences with pairwise alignments. Bioinformatics 2005, 21 :282-292.

12. Firth $A E$, Brown $C M$ : Detecting overlapping coding sequences in virus genomes. BMC Bioinformatics 2006, 7:75.

13. Chung BYW, Miller WA, Atkins JF, Firth AE: An overlapping essential gene in the Potyviridae. Proc Natl Acad Sci USA 2008, 1 05:5897-5902.

14. Firth AE, Chung BY, Fleeton MN, Atkins JF: Discovery of frameshifting in Alphavirus $6 \mathrm{~K}$ resolves a 20 -year enigma. Virol J 2008, 5: 108.

15. Firth AE, Atkins JF: A conserved predicted pseudoknot in the NS2A-encoding sequence of West Nile and Japanese encephalitis flaviviruses suggests NSI' may derive from ribosomal frameshifting. Virol | 2009, 6:|4

16. Altschul SF, Gish W, Miller W, Myers EW, Lipman DJ: Basic local alignment search tool. J Mol Biol 1990, 2 I 5:403-4I 0 .

17. Thompson JD, Higgins DG, Gibson TJ: CLUSTAL W: improving the sensitivity of progressive multiple sequence alignment through sequence weighting, position-specific gap penalties and weight matrix choice. Nucleic Acids Res 1994, 22:4673-4680.

18. Wijegoonawardane PK, Sittidilokratna N, Petchampai N, Cowley JA, Gudkovs N, Walker PJ: Homologous genetic recombination in the yellow head complex of nidoviruses infecting Penaeus monodon shrimp. Virology 2009, 390:79-88

19. Matsuda D, Dreher TW: Close spacing of AUG initiation codons confers dicistronic character on a eukaryotic mRNA. RNA 2006, I 2: I338-1349.

20. Belshaw R, Pybus OG, Rambaut A: The evolution of genome compression and genomic novelty in RNA viruses. Genome Res 2007, I 7:| 496-I504.

2I. Rancurel C, Khosravi M, Dunker KA, Romero PR, Karlin D: Overlapping genes produce proteins with unusual sequence properties and offer insight into de novo protein creation. J Virol 2009, 83:10719-10736.

22. Zdobnov EM, Apweiler R: InterProScan - an integration platform for the signature-recognition methods in InterPro. Bioinformatics 2001, 1 7:847-848.

23. Bendtsen JD, Nielsen H, von Heijne G, Brunak S: Improved prediction of signal peptides: SignalP 3.0. J Mol Biol 2004, 340:783-795.

Publish with Biomed Central and every scientist can read your work free of charge

"BioMed Central will be the most significant development for disseminating the results of biomedical research in our lifetime. "

Sir Paul Nurse, Cancer Research UK

Your research papers will be:

- available free of charge to the entire biomedical community

- peer reviewed and published immediately upon acceptance

- cited in PubMed and archived on PubMed Central

- yours - you keep the copyright 